\title{
Tuning domain wall velocity with Dzyaloshinskii-Moriya interaction
}

\author{
Fernando Ajejas, ${ }^{1,2}$ Viola Křižáková, ${ }^{3}$ Dayane de Souza Chaves, ${ }^{3}$ Jan Vogel, ${ }^{3}$ Paolo Perna, ${ }^{2}$ Ruben Guerrero, ${ }^{2}$ \\ Adrian Gudin, ${ }^{2}$ Julio Camarero, ${ }^{1,2}$ and Stefania Pizzini ${ }^{3}$ \\ 1) Universidad Autonoma de Madrid, Madrid, Spain \\ 2) IMDEA Nanoscience, Campus de Cantoblanco, 28049 Madrid, Spain \\ ${ }^{3)}$ Univ. Grenoble Alpes, CNRS, Institut Néel, F-38000 Grenoble, France
}

(Dated: 29 September 2017)

\begin{abstract}
We have studied a series of $\mathrm{Pt} / \mathrm{Co} / \mathrm{M}$ epitaxial trilayers, in which $\mathrm{Co}$ is sandwiched between $\mathrm{Pt}$ and a non magnetic layer $\mathrm{M}(\mathrm{Pt}, \mathrm{Ir}, \mathrm{Cu}, \mathrm{Al})$. Using polar magneto-optical Kerr microscopy, we show that the fieldinduced domain wall speeds are strongly dependent on the nature of the top layer, they increase going from $\mathrm{M}=\mathrm{Pt}$ to lighter top metallic overlayers, and can reach several $100 \mathrm{~m} / \mathrm{s}$ for $\mathrm{Pt} / \mathrm{Co} / \mathrm{Al}$. The $\mathrm{DW}$ dynamics is consistent with the presence of chiral Néel walls stabilized by interfacial Dzyaloshinskii-Moriya interaction (DMI) whose strength increases going from $\mathrm{Pt}$ to $\mathrm{Al}$ top layers. This is explained by the presence of DMI with opposite sign at the $\mathrm{Pt} / \mathrm{Co}$ and $\mathrm{Co} / \mathrm{M}$ interfaces, the latter increasing in strength going towards heavier atoms, possibly due to the increasing spin-orbit interaction. This work shows that in non-centrosymmetric trilayers the domain wall dynamics can be finely tuned by engineering the DMI strength, in view of efficient devices for logic and spitronics applications.
\end{abstract}

Asymmetric magnetic stacks in which a thin magnetic layer is sandwiched between two heavy metals or a heavy metal and an oxide, hold promise for applications in the field of spintronics or spinorbitronics. They can host chiral domain walls ${ }^{1}$ and skyrmions ${ }^{2}$ that can be displaced efficiently with fields or current pulses ${ }^{3-5}$, so that they are envisaged as carriers of binary information in logic devices and racetrack memories ${ }^{6}$. The essential ingredient necessary to stabilize such objects is a strong interfacial Dzyaloshinskii-Moriya interaction ${ }^{7,8}$, an antisymmetric exchange energy term in competition with Heisenberg exchange that can lead to spiral magnetic structures with a defined chirality.

In order to optimize materials for efficient transfer of digital information, the DMI strength should be controlled and possibly tuned. Nowadays, little is known about the microscopic origins of such interfacial interaction and how it changes as a function of the $3 d$ or the $5 d$ metal at $3 d / 5 d$ interfaces. Some ab initio calculations were performed to address quantitatively the DMI in several HM/FM bilayer systems ${ }^{9-11}$ and models have been proposed to explain the DMI variation for several $3 d / 5 d$ interfaces ${ }^{11,12}$. These calculations can guide the optimization of materials with large DMI, although ab initio calculations consider ideal interfaces, while experiments concentrate on samples generally grown by magnetron sputtering, where interfacial structure and quality influences the strength and even the sign of the $\mathrm{DMI}^{13}$. For these reasons, it is rare that experimental findings match the theoretical predictions.

The $\mathrm{Pt} / \mathrm{Co}$ interface is the prototypical interface hosting a large DMI. Theoretical ${ }^{9,10,14}$ and experimental studies $^{15-18}$ agree on the fact that it is the source of strong DMI favoring homochiral magnetic textures (chiral Néel walls and skyrmions) with anticlockwise rotation of the magnetic moments (left-handed chirality). Very little is known about the DMI at interfaces where Co is in contact with other heavy metals. Only the $\mathrm{Pt} / \mathrm{Co} / \mathrm{Ir}$ stack has been largely studied, following the $a b$ initio calculations by Yang et al. that predicted opposite signs for the DMI at $\mathrm{Pt}(111) / \mathrm{Co}$ and $\operatorname{Ir}(111) / \mathrm{Co}$ interfaces ${ }^{10}$. However, experimental data do not allow concluding on this matter. Moreau-Luchaire et al. ${ }^{19}$ reported high DMI values for $\mathrm{Pt} / \mathrm{Co} / \mathrm{Ir}$ multilayers, that they attributed to the same sign of the DMI at $\mathrm{Pt} / \mathrm{Co}$ and $\mathrm{Co} / \mathrm{Ir}$ interfaces. The work of Chen et al..$^{20}$ and that of Hrabec et $a l .{ }^{21}$ also agree with the theoretical prediction. On the other hand Kim et al. ${ }^{18}$ show the same sign of the $\mathrm{DMI}$ in $\mathrm{Pt} / \mathrm{Co} / \mathrm{AlOx}$ and $\mathrm{Ir} / \mathrm{Co} / \mathrm{AlOx}$ trilayers, while Han et al. ${ }^{22}$ found lower DMI for $\mathrm{Pt} / \mathrm{Co} / \mathrm{Ir}$ compared to $\mathrm{Pt} / \mathrm{Co} / \mathrm{AlOx}$ and suggested that this might be due to the opposite DMI signs at $\mathrm{Pt} / \mathrm{Co}$ and $\mathrm{Co} / \mathrm{Ir}$ interfaces. A consensus is still missing on this matter and the contradictory results may be due to the different morphology of the interface between Co and heavy metal in the various materials.

In this work we address the study of DMI in epitaxial layers with well defined (111) texture, like that considered in the $a b$ initio work of Ref. ${ }^{10}$. The DMI of $\mathrm{Pt} / \mathrm{Co} / \mathrm{M}$ trilayers with different metallic overlayers $(\mathrm{M}=\mathrm{Al}, \mathrm{Cu}, \mathrm{Ir}, \mathrm{Pt})$ was studied through the measurement of domain wall dynamics. We show that the DMI at the $\mathrm{Co} / \mathrm{M}$ interface has the same sign for all the studied overlayers, with increasing strength as the atomic number increases. The domain wall speed in Co is strongly dependent on the DMI strength, the largest speed being obtained for $\mathrm{Pt} / \mathrm{Co} / \mathrm{Al}$, the smallest for $\mathrm{Pt} / \mathrm{Co} / \mathrm{Pt}$ where the DMI at the two Co interfaces compensate. This finding may be useful for engineering samples with optimized DMI for spintronic applications.

The $\mathrm{Pt} / \mathrm{Co} / \mathrm{M}$ (with $\mathrm{M}=\mathrm{Al}, \mathrm{Cu}$, Ir, $\mathrm{Pt}$ ) epitaxial stacks were grown on commercial $\mathrm{MgO}(111)$ single crystals. The morphology of the (111) surface was improved by annealing at high temperature. After one hour of heat treatment at $450^{\circ} \mathrm{C}, 28 \mathrm{~nm}$ of $\mathrm{Pt}$ were deposited by dc magnetron sputtering using $6 \times 10^{-3}$ mbar $\mathrm{Ar}^{+}$pressure and $20 \mathrm{~W}$ magnetron power. The layer thickness was calibrated by a quartz balance in- 


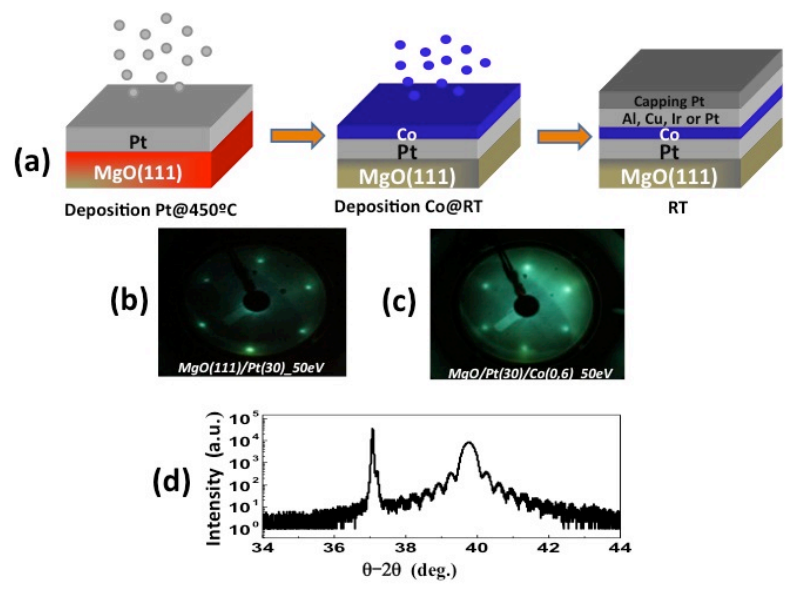

FIG. 1. (a) Sketch of the sample growth process; (b) LEED patterns obtained after the growth of the Pt underlayer; (c) LEED pattern after the growth of the Co layer; (d) XRD pattern of the $\mathrm{Pt} / \mathrm{Co} / \mathrm{Pt}$ trilayer.

stalled in the sputtering chamber. The surface quality was checked in-situ by LEED. The LEED patterns acquired for $\mathrm{MgO}(111) / \mathrm{Pt}$ indicate good surface quality with hexagonal reconstruction (Figure 1). The XRD scans covering the region of the FCC Pt(111) crystallographic peak clearly shows that only a single-phase FCC with [111] orientation is present, with no other structural domains coexisting. The $\sim 0.6 \mathrm{~nm}$-thick Co, the 2nm-thick $M$ layers and the 2nm-thick Pt capping were grown in general at RT, in order to avoid intermixing at the interfaces. The (111) texture is kept after the Co deposition (Figure 1). For some of the samples, the Co and $\mathrm{M}$ layers were grown at $100^{\circ} \mathrm{C}$. We also compared $\mathrm{Pt}(28 \mathrm{~nm}) / \mathrm{Ir}(2 \mathrm{~nm}) / \mathrm{Co}(0.6 \mathrm{~nm}) / \mathrm{Pt}(2 \mathrm{~nm})$ and $\mathrm{Pt}(30 \mathrm{~nm}) / \mathrm{Co}(0.6) / \operatorname{Ir}(2 \mathrm{~nm}) / \mathrm{Pt}(2 \mathrm{~nm})$ samples to check the influence of inversion of the structure on the DMI. The magnetic properties were measured with VSMSQUID and magneto-optical Kerr effect. All the samples present perpendicular magnetic anisotropy (PMA) with square hysteresis loops. The unit surface magnetization $M_{s} t$ ( $t$ being the thickness of the Co layer) and the in-plane saturation field $\mu_{0} H_{K}$ are reported in Table 1.

The domain wall dynamics was studied using wide field magneto-optical Kerr microscopy. The DW velocity was deduced from the expansion of bubble domains, driven by out-of-plane magnetic field pulses $H_{z}$ of strength up to $600 \mathrm{mT}$, obtained with microcoils associated to pulsed current generators.

The sign and the strength of the DMI for the $\mathrm{Pt} / \mathrm{Co} / \mathrm{M}$ samples was extracted by studying the domain wall expansion driven by $H_{z}$ pulses, in the presence of a constant in-plane magnetic field $H_{x}$ parallel to the DW normal $^{21,23-25}$. In systems with DMI and chiral Néel walls, the DW propagation is anisotropic in the direction of $H_{x}$ and the DW speed is larger for the domain walls having magnetization parallel to $H_{x}$. This allows us

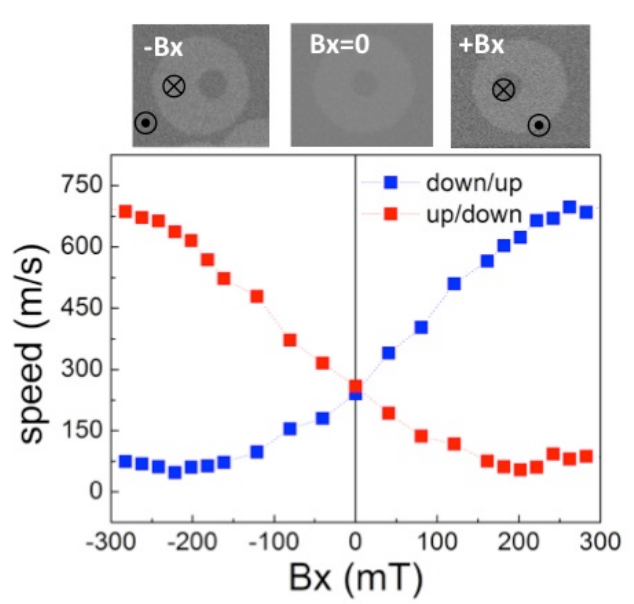

FIG. 2. (Top): Differential Kerr images showing the asymmetric propagation of up/down and down/up DWs in the direction of the $B_{x}$ field in $\mathrm{Pt} / \mathrm{Co} / \mathrm{Al}$, compared with the isotropic propagation when $B_{x}=0$. (Bottom): Domain wall speed vs. $B_{x}$ for up/down and down/up DWs propagating in the x-direction. The driving field is $B_{z}=300 \mathrm{mT}$.

to obtain without ambiguity the chirality of the domain walls. The DMI strength can be obtained from the $H_{z^{-}}$ driven DW speed vs. the intensity of $H_{x}$. In the presence of DMI, the DW speed reaches a minimum when $H_{x}$ compensates the $H_{D M I}$ field that stabilizes the Néel walls. From the value of this field we can then deduce the average DMI energy density $D$, since $H_{D M I}=D /\left(\mu_{0} M_{s} \Delta\right)$ where $\Delta=\sqrt{A / K_{0}}, A$ is the exchange stiffness and $K_{0}$ the effective anisotropy energy. The $H_{z}$ field driving the DWs was chosen to be beyond the depinning field, giving rise to a reliable measurement of the $\mathrm{DMI}^{24,25}$.

Figures 2 and 3 present the differential Kerr images showing the anisotropic expansion of bubble domains driven by an $H_{z}$ pulse in the presence of an $H_{x}$ field, measured for $\mathrm{Pt} / \mathrm{Co} / \mathrm{Al}, \mathrm{Pt} / \mathrm{Co} / \mathrm{Ir}$ and $\mathrm{Ir} / \mathrm{Co} / \mathrm{Pt}$ stacks. The same behaviour was found for $\mathrm{Pt} / \mathrm{Co} / \mathrm{Cu}$ trilayers, as expected for systems with chiral Néel walls. On the other hand, in $\mathrm{Pt} / \mathrm{Co} / \mathrm{Pt}$ stack the DW expansion is perfectly isotropic in the sample plane, as also found for polycrystalline $\mathrm{Pt} / \mathrm{Co} / \mathrm{Pt}$ samples $^{25}$. This is consistent with the absence of inversion asymmetry, that leads to negligible DMI and to the stabilization of achiral Bloch walls.

Note that the down/up domain walls propagate with larger velocity for positive $H_{x}$ fields for $\mathrm{Pt} / \mathrm{Co} / \mathrm{M}$ with $\mathrm{M}=\mathrm{Al}, \mathrm{Cu}$, Ir but with smaller velocity for $\mathrm{Ir} / \mathrm{Co} / \mathrm{Pt}$. These measurements are consistent with the presence of left handed chirality for the domain walls in $\mathrm{Pt} / \mathrm{Co} / \mathrm{Al}$, $\mathrm{Pt} / \mathrm{Co} / \mathrm{Cu}$ and $\mathrm{Pt} / \mathrm{Co} / \mathrm{Ir}$, and an opposite, right-handed, chirality for DWs in Ir/Co/Pt. This is expected using symmetry arguments, as the layer sequence is inverted with respect to the growth axis along which the symmetry is broken.

Figures 2 and 3 also show that the speed of up/down and down/up DWs measured vs. the $H_{x}$ intensity reaches 
TABLE I. Effective anisotropy field $\mu_{0} H_{K}$, unit surface magnetization $M_{s} t$, saturation domain wall speed $v_{m a x}$, DMI field $\mu_{0} H_{D M I}$, effective interface DMI energy density extracted from $v_{\max }\left(\mathrm{D}_{s}{ }^{e f f, v}\right.$ ) and from the DMI field $\left(\mathrm{D}_{s}{ }^{e f f, H}\right), \mathrm{DMI}$ energy density at the $\mathrm{Pt} / \mathrm{Co}$ interface $\left(\mathrm{D}_{s}{ }^{P t / C o}\right)$ and at the $\mathrm{Co} / \mathrm{M}$ interface $\left(\mathrm{D}_{s}{ }^{C o / M}\right)$ for the Pt/Co/M stacks. Positive DMI values in the table stand for left-handed DW chiralities.

\begin{tabular}{|c|c|c|c|c|c|c|c|c|c|}
\hline Sample & $\begin{array}{l}\mathrm{T}_{C o} \\
\left({ }^{\circ} \mathrm{C}\right)\end{array}$ & $\begin{array}{l}\mu_{0} \mathrm{H}_{K} \\
(\mathrm{~T})\end{array}$ & $\begin{array}{l}\mathrm{M}_{s} \mathrm{t} \\
(\mathrm{mA})\end{array}$ & $\begin{array}{l}\mathrm{v}_{\max } \\
(\mathrm{m} / \mathrm{s})\end{array}$ & $\begin{array}{l}\mu_{0} \mathrm{H}_{D M I} \\
(\mathrm{mT})\end{array}$ & $\begin{array}{l}\mathrm{D}_{s}^{e f f, v} \\
(\mathrm{pJ} / \mathrm{m})\end{array}$ & $\begin{array}{l}\mathrm{D}_{s}^{e f f, H} \\
(\mathrm{pJ} / \mathrm{m})\end{array}$ & $\begin{array}{l}\mathrm{D}_{s}^{P t / C o} \\
(\mathrm{pJ} / \mathrm{m})\end{array}$ & $\begin{array}{l}\mathrm{D}_{s}{ }^{C o / M} \\
(\mathrm{pJ} / \mathrm{m})\end{array}$ \\
\hline $\mathrm{Pt} / \mathrm{Co} / \mathrm{Al}$ & $\mathrm{RT}$ & $1.3 \pm 0.1$ & $1.3 \pm 0.1$ & $280 \pm 10$ & $212 \pm 10$ & $1.32 \pm 0.1$ & $1.21 \pm 0.1$ & $1.26 \pm 0.1$ & 0 \\
\hline $\mathrm{Pt} / \mathrm{Co} / \mathrm{Al}$ & 100 & 1.8 & 0.92 & 250 & 200 & 0.83 & 0.91 & 0.87 & 0 \\
\hline $\mathrm{Pt} / \mathrm{Co} / \mathrm{Ir}$ & $\mathrm{RT}$ & 1.4 & 0.75 & 80 & 96 & 0.22 & 0.30 & 1.26 & -1.0 \\
\hline $\mathrm{Pt} / \mathrm{Co} / \mathrm{Ir}$ & 100 & 1.3 & 1.0 & 115 & 125 & 0.40 & 0.55 & 0.87 & -0.39 \\
\hline $\mathrm{Ir} / \mathrm{Co} / \mathrm{Pt}$ & $\mathrm{RT}$ & 0.6 & 0.9 & 200 & 106 & -0.65 & -0.65 & & \\
\hline $\mathrm{Pt} / \mathrm{Co} / \mathrm{Cu}$ & 100 & 1.8 & 0.75 & 230 & 200 & 0.62 & 0.56 & 0.87 & -0.27 \\
\hline $\mathrm{Pt} / \mathrm{Co} / \mathrm{Pt}$ & 100 & 1.2 & 1.3 & 20 & 0.00 & - & - & 0.87 & -0.87 \\
\hline
\end{tabular}
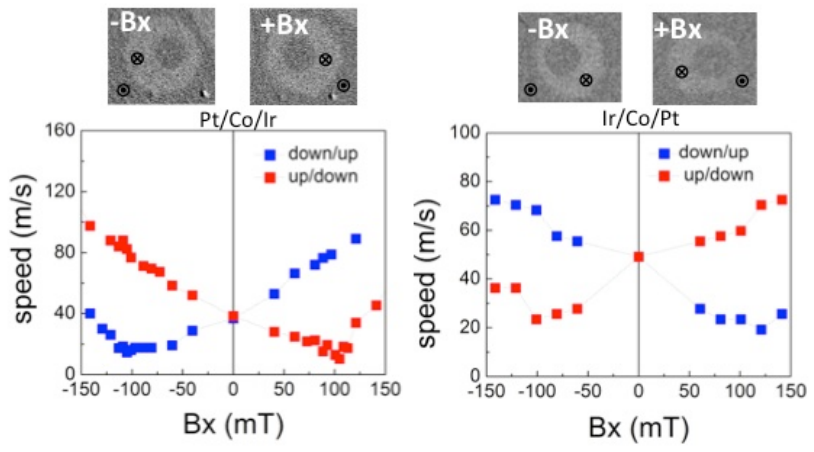

FIG. 3. (Top) Differential Kerr images showing the asymmetric propagation of domain walls in $\mathrm{Pt} / \mathrm{Co} / \mathrm{Ir}$ (left) and $\mathrm{Ir} / \mathrm{Co} / \mathrm{Pt}$ (right), with a driving field $B_{z}$ around $290 \mathrm{mT}$ and an in-plane field $B_{x}$ around $100 \mathrm{mT}$. (Bottom): speed vs. $B_{x}$ for up/down and down/up DWs propagating in the $\mathrm{x}$-direction. Note the opposite behaviour of up/down and down/up DWs in the two samples, demonstrating the opposite chirality of the DWs.

a minimum, for a field corresponding to the in-plane field compensating $H_{D M I}$. The strength of the interfacial DMI strength $D_{s}=D t$ (in $\mathrm{pJ} / \mathrm{m}$ ) was obtained from the expression of the DMI field given above, using the measured magnetic parameters and exchange stiffness $A=16$ $\mathrm{pJ} / \mathrm{m}$.

The DMI strengths obtained for the various samples are shown in Table 1 together with the measured magnetic parameters. Data are shown for samples with Co grown both at RT and at $100^{\circ} \mathrm{C}$. Let us first look at the DMI values for Co grown at RT. The largest value of the DMI is obtained for $\mathrm{Pt} / \mathrm{Co} / \mathrm{Al}\left(D_{s} e^{\text {eff }}=1.2-1.3 \mathrm{pJ} / \mathrm{m}\right)$, while it falls to $D_{s}{ }^{e f f}=0.2-0.3 \mathrm{pJ} / \mathrm{m}$ for $\mathrm{Pt} / \mathrm{Co} / \mathrm{Ir}$ and is vanishing for $\mathrm{Pt} / \mathrm{Co} / \mathrm{Pt}$. If we consider that the two Co interfaces are sufficiently apart so that they give a distinct contribution to the $\mathrm{DMI}^{10}$, then $D_{s}$ eff $=$ $D_{s}{ }^{P t / C o / M}=D_{s}{ }^{P t / C o}+D_{s}{ }^{C o / M}$. Since for all the samples the growth process was identical up to the Co layer, we can assume that the DMI strength at the Pt/Co inter- face does not change from one sample to another. These results then indicate that the DMI decrease going from $\mathrm{Al}$ to $\mathrm{Ir}$ and to $\mathrm{Pt}$, is due to the consequent increase of the contribution of the $\mathrm{Co} / \mathrm{M}$ interface, its sign being opposite to that of the $\mathrm{Pt} / \mathrm{Co}$ interface. Since Al has a low atomic number and therefore a weak SOC, we suppose that the $\mathrm{DMI}$ at the $\mathrm{Co} / \mathrm{Al}$ interface is negligible and that the $\mathrm{DMI}$ of the $\mathrm{Pt} / \mathrm{Co} / \mathrm{Al}$ trilayer is concentrated at the $\mathrm{Pt} / \mathrm{Co}$ interface. This is corroborated by our recent measurements of a series of $\mathrm{Pt} / \mathrm{Co} / \mathrm{AlOx}$ samples with variable oxygen concentration at the top Co interface ${ }^{26}$. While the under-oxidized top interface, where Co is essentially in contact with $\mathrm{Al}$, does not contribute to the DMI and to the PMA, a gradual increase of the DMI occurs as the $\mathrm{Al}$ atoms are substituted by $\mathrm{O}$. The value of the interfacial DMI for the under-oxidized $\mathrm{Pt} / \mathrm{Co} / \mathrm{AlOx}$ sample was found to be around $1.2 \mathrm{pJ} / \mathrm{m}$ in agreement with the results reported here.

Using these arguments, our results suggest that the small value of the DMI in the $\mathrm{Pt} / \mathrm{Co} / \mathrm{Ir}$ trilayer is due to a large DMI contribution of the $\mathrm{Co} / \mathrm{Ir}$ interface $\left(D_{s}{ }^{C o / I r}=-1 \mathrm{pJ} / \mathrm{m}\right)$, opposite to that of the Pt/Co interface $\left.D_{s}{ }^{P t / C o}=1.2-1.3 \mathrm{pJ} / \mathrm{m}\right)$. This is in agreement with the results of Kim et al. ${ }^{18}$ who find the same DMI sign at $\mathrm{Pt} / \mathrm{Co}$ and $\mathrm{Ir} / \mathrm{Co}$ interfaces in polycrystalline $\mathrm{HM} / \mathrm{Co} / \mathrm{AlOx}(\mathrm{HM}=\mathrm{Pt}$, Ir $)$ trilayers. In their case the DMI at the Ir/Co interface is a factor 3 smaller than that of the Pt/Co interface: this may be attributed to the different details of the HM/Co interface morphology, which can strongly influence the DMI. The better crystallinity of our samples may explain the better "compensation" of the DMI at the two interfaces (larger DMI at Co/Ir), which is realized perfectly in the case of $\mathrm{Pt} / \mathrm{Co} / \mathrm{Pt}$.

Note that for $\mathrm{Pt} / \mathrm{Co} / \mathrm{Al}$, the DMI decreases to $D_{s}{ }^{e f f} \sim 0.9 \mathrm{pJ} / \mathrm{m}$ when the Co layer is grown at $100^{\circ} \mathrm{C}$. Since the $\mathrm{Co} / \mathrm{Al}$ interface does not give rise to $\mathrm{DMI}$, this deterioration of the DMI is probably due to the slight interdiffusion at the $\mathrm{Pt} / \mathrm{Co}$ interface. A change occurs also for the DMI of $\mathrm{Pt} / \mathrm{Co} / \mathrm{Ir}$ grown at high temperature, but in this case the effective DMI increases, as the different modification of the DMI at the two interfaces gives rise to a worse compensation. Assuming again that 


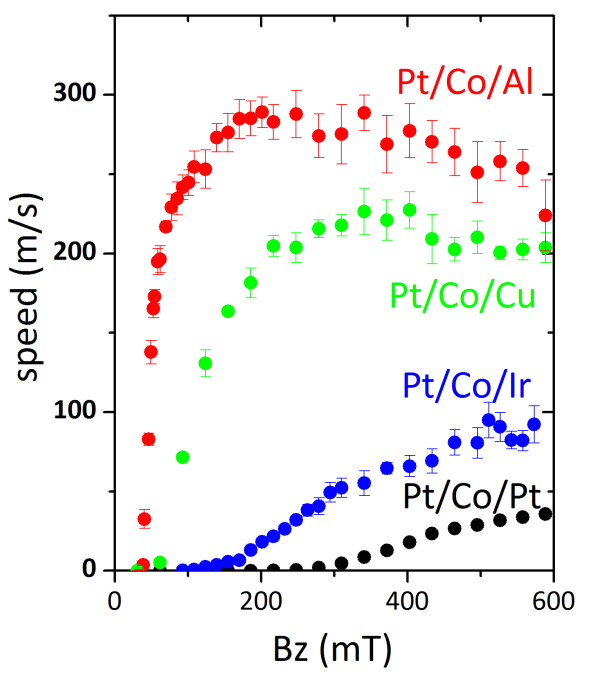

FIG. 4. (a) Domain wall speed vs. out-of-plane magnetic field, measured for $\mathrm{Pt} / \mathrm{Co} / \mathrm{Pt}, \mathrm{Pt} / \mathrm{Co} / \mathrm{Ir}, \mathrm{Pt} / \mathrm{Co} / \mathrm{Cu}$ and $\mathrm{Pt} / \mathrm{Co} / \mathrm{Al}$. Note the increasing saturation speed as the DMI gets larger.

$D_{s}^{P t / C o} \sim 0.9 \mathrm{pJ} / \mathrm{m}$ also in this trilayer, then the DMI at the $\mathrm{Co} / \mathrm{Ir}$ is shown to decrease to $D_{s}{ }^{C o} / \mathrm{Ir} \sim-0.4 \mathrm{pJ} / \mathrm{m}$ when $\mathrm{Co}$ is grown at high temperature. These results show that the morphology of the interface is as expected an important parameter that can strongly influence the strength of the DMI (see also ${ }^{13}$ ).

The $\mathrm{Pt} / \mathrm{Co} / \mathrm{Cu}$ sample has an intermediate behavior between $\mathrm{Pt} / \mathrm{Co} / \mathrm{Al}$ and $\mathrm{Pt} / \mathrm{Co} / \mathrm{Ir}$. For technical reasons only the sample with Co grown at $100^{\circ} \mathrm{C}$ could be measured, for which we found $D_{s}{ }^{e f f} \sim 0.6 \mathrm{pJ} / \mathrm{m}$. Using the same assumption for the $\mathrm{Pt} / \mathrm{Co}$ interface, we deduce that $D_{s}^{C o / C u} \sim-0.3 \mathrm{pJ} / \mathrm{m}$, that is smaller than the value obtained for the $\mathrm{Co} / \mathrm{Ir}$ interface grown at the same temperature. Since $\mathrm{Co}$ and $\mathrm{Cu}$ are immiscible at $100^{\circ} \mathrm{C}$, we do not expect a large variation of this value for a sample with Co grown at RT.

The observed increase of the DMI at the $\mathrm{Co} / \mathrm{M}$ interface, with $\mathrm{M}$ going from $\mathrm{Al}$ to $\mathrm{Cu}$ to $\mathrm{Ir}$ and finally to $\mathrm{Pt}$ may be related to the increasing atomic number and therefore to the increasing SOC, which is known to be at the origin of the DMI.

These results show that the DMI of these stacks can be easily manipulated and controlled by varying the chemical nature of the top layer. This can provide an interesting step towards the realization of ad hoc materials for spintronic devices, in which the DMI is an essential parameter e.g. to stabilize magnetic skyrmions.

We also investigated the effect of the effective DMI variation on the domain wall velocity vs. out-of-plane field. This is shown in Figure 4. The DW dynamics strongly depends on the chemical nature of the top layer, the largest velocity obtained for $\mathrm{Pt} / \mathrm{Co} / \mathrm{Al}$ (maximum DMI) being a factor 10 larger than for $\mathrm{Pt} / \mathrm{Co} / \mathrm{Pt}$ (vanishing DMI) in the same field range. This result is related to the fact that in samples with DMI the saturation DW velocity is linearly proportional to $D_{S}$, as $v_{\max }=\frac{\pi}{2} \gamma \frac{D_{s}}{M_{s} t} 25,26$. Table 1 shows the values of the interfacial DMI found using this analytical expression and the experimental $v_{\max }$ and unit surface magnetization $M_{s} t$. These values are in excellent agreement with those found with the method used above.

In conclusion, we have shown that the DMI and, as a consequence, the domain wall velocity in $\mathrm{Pt} / \mathrm{Co} / \mathrm{M}$ trilayers can be tuned by changing the chemical species of the top metallic layer. This provides an interesting way to tune the DMI of trilayer system in view of optimized spintronics or logic devices.

In these epitaxial samples with strong (111) texture, the DMI at the $\mathrm{Co} / \mathrm{M}$ interface (with $\mathrm{M}=\mathrm{Al}, \mathrm{Cu}$, Ir and $\mathrm{Pt}$ ) was found to have opposite sign to that of the $\mathrm{Pt} / \mathrm{Co}$ interface, and to increase in strength for heavier atoms, probably because of the increasing SOC. Since the effective DMI is the sum of the DMI at top and bottom Co interfaces, its maximum strength (and maximum $\mathrm{DW}$ speed) is obtained for $\mathrm{Pt} / \mathrm{Co} / \mathrm{Al}$ trilayers where the $\mathrm{Co} / \mathrm{Al}$ interface gives a negligible contribution to the DMI. The microscopic reasons for the disagreement between these measurements and the results of $a b$ initio calculations are still to be revealed, but our results point out the importance of the details of the interface structure and its effects on the electronic hybridization.

Preliminary data on $\mathrm{Co} / \mathrm{Ta}$ and $\mathrm{Co} / \mathrm{W}$ layers grown on $\mathrm{Pt}(111)$ suggest a DMI lower and of opposite sign of that of the $\mathrm{Pt} / \mathrm{Co}$ interface, like for $\mathrm{Co} / \mathrm{Al}, \mathrm{Co} / \mathrm{Cu}$ and $\mathrm{Co} / \mathrm{Ir}$ interfaces. These data suggest that among the interfaces investigated to date, the $\mathrm{Pt} / \mathrm{Co}$ is the one providing the largest DMI. An efficient way to increase the DMI of a complete trilayer, is to cover the Co layer with an optimized concentration of oxygen ${ }^{26}$.

\section{ACKNOWLEDGMENTS}

S. P. and J. V. acknowledge the support of the Agence Nationale de la Recherche, project ANR-14-CE26-0012 (ULTRASKY). B. Fernandez, Ph. David and E. Mossang are acknowledged for their technical help. D.S.C was supported by a CNPq Scholarship (Brazil). F. A. acknowledges the support of Spanish MINECO for exchange grant EEBB-I-16-11844. P.P., R.G., A.G. and J.C. acknowledge the support of Spanish MINECO project FIS2016-78591-C3-1-R (SKYTRON). F.A., P.P. and J.C. acknowledge the support of the Comunidad de Madrid through project NANOFRONTMAG CM.

${ }^{1}$ A. Thiaville, S. Rohart, E. Jué, V. Cros, and A. Fert, EPL 100, 57002 (2012).

${ }^{2}$ T. Skyrme, Nucl. Phys. 31, 556 (1962).

${ }^{3}$ K.-S. Ryu, T. L., S.-H. Yang, and S. Parkin, Nat. Nanotech. 8, 527 (2013).

${ }^{4}$ S. Emori, U. Bauer, S.-M. Ahn, E. Martinez, and G. Beach, Nat. Mater. 12, 611 (2013).

${ }^{5}$ A. Fert, V. Cros, and N. Reyren, Nature Rev. Mater. 2, 17031 (2017). 
${ }^{6}$ S. Parkin, M. Hayashi, and L. Thomas, Science 320, 190 (2008).

${ }^{7}$ I. E. Dzyaloshinskii, Sov. Phys. JETP 5, 1259 (1957).

${ }^{8}$ T. Moriya, Phys. Rev. 120, 91 (1960).

${ }^{9}$ F. Freimuth, S. Blügel, and Y. Mokrousov, J. Phys.: Condens. Matter 26, 104202 (2014).

${ }^{10}$ H. Yang, A. Thiaville, S. Rohart, A. Fert, and M. Chshiev, Phys. Rev. Lett. 115, 267210 (2015).

${ }^{11}$ A. Belabbes, S. Blügel, and A. Manchon, Phys. Rev. Lett. 117, $247202(2016)$.

${ }^{12}$ V. Kashid, T. Schena, B. Zimmermann, Y. Mokrousov, S. Blügel, V. Shah, and H. G. Salunke, Phys. Rev. B 90, 054412 (2014).

${ }^{13}$ A. W. J. Wells, P. M. Shepley, C. H. Marrows, and T. A. Moore, Phys. Rev. B 95, 054428 (2017).

${ }^{14} \mathrm{H}$. Yang, O. Boulle, V. Cros, A. Fert, and M. Chshiev, arXiv:1603.01847 (2016).

${ }^{15}$ S. Pizzini, J. Vogel, S. Rohart, L. Buda-Prejbeanu, E. Jué, O. Boulle, I. Miron, C. Safeer, S. Auffret, G. Gaudin, and A. Thiaville, Phys. Rev. Lett. 113, 047203 (2014).

${ }^{16}$ M. Belmeguenai, J.-P. Adam, Y. Roussigné, S. Eimer, T. Devolder, J.-V. Kim, S. Cherif, A. Stashkevich, and A. Thiaville, Phys. Rev. B 91, 180405(R) (2015).

${ }^{17}$ J. Cho, N.-H. Kim, S. Lee, J.-S. Kim, R. Lavrijsen, A. Solignac, Y. Yin, D.-S. Han, N. J. J. van Hoof, H. J. M. Swagten, B. Koopmans, and C.-Y. You, Nature Comm. 6 (2015).

${ }^{18}$ N.-H. Kim, J. Jung, J. Cho, D.-S. Han, Y. Yin, J.-S. Kim,
H. J. M. Swagten, and C.-Y. You, Applied Physics Letters 108, 142406 (2016), http://dx.doi.org/10.1063/1.4945685.

${ }^{19}$ C. Moreau-Luchaire, C. Moutafis, N. Reyren, J. Sampaio, C. A. F. Vaz, N. Van Horne, K. Bouzehouane, K. Garcia, C. Deranlot, P. Warnicke, P. Wohlhuter, J. M. George, M. Weigand, J. Raabe, V. Cros, and A. Fert, Nature Nanotechn. 11, 444 (2016).

${ }^{20}$ G. Chen, T. Ma, A. T. N'Diaye, H. Kwon, C. Won, Y. Wu, and A. K. Schmid, Nat. Commun. 4, 2671 (2013).

${ }^{21}$ A. Hrabec, N. A. Porter, A. Wells, M. J. Benitez, G. Burnell, S. McVitie, D. McGrouther, T. A. Moore, and C. H. Marrows, Phys. Rev. B 90, 020402 (2014).

${ }^{22}$ D.-S. Han, N.-H. Kim, J.-S. Kim, Y. Yin, J.-W. Koo, J. Cho, S. Lee, M. Klui, H. J. M. Swagten, B. Koopmans, and C.-Y. You, Nano Letters 16, 4438 (2016).

${ }^{23}$ S.-G. Je, D.-H. Kim, S.-C. Yoo, B.-C. Min, K.-J. Lee, and S.-B. Choe, Phys. Rev. B 88, 214401 (2013).

${ }^{24}$ M. Vaňatka, J.-C. Rojas-Sánchez, J. Vogel, M. Bonfim, A. Thiaville, and S. Pizzini, J. Phys.: Condens. Matter 27, 32002 (2015).

${ }^{25}$ T. H. Pham, J. Vogel, J. Sampaio, M. Vaňatka, J.-C. RojasSanchez, M. Bonfim, D. S. Chaves, F. Choueikani, P. Ohresser, E. Otero, A. Thiaville, and S. Pizzini, EPL 113, 67001 (2016).

${ }^{26}$ D. Chaves, F. Ajejas, V. Krizakova, J. Vogel, and S. Pizzini, arXiv:1708.08516 (2017). 